\title{
The Fate of Partially Thrombosed Intracranial Aneurysms Treated with Endovascular Intervention
}

\author{
Jeongjun Lee, ${ }^{1}$ Won-Sang Cho, ${ }^{2}$ Roh Eul Yoo, ${ }^{3}$ Dong Hyun Yoo, ${ }^{3}$ Young Dae Cho, ${ }^{3}$ Hyun-Seung Kang, ${ }^{2}$ Jeong Eun Kim ${ }^{2}$ \\ Department of Neurosurgery, Dongguk University Ilsan Hospital, Goyang, Korea \\ Department of Neurosurgery, ${ }^{2}$ Seoul National University Hospital, Seoul, Korea \\ Department of Radiology, ${ }^{3}$ Seoul National University Hospital, Seoul, Korea
}

Objective : The fate of partially thrombosed intracranial aneurysms (PTIAs) is not well known after endovascular treatment. The authors aimed to analyze the treatment outcomes of PTIAs.

Methods : We retrospectively reviewed the medical records of 27 PTIAs treated with endovascular intervention between January 1999 and March 2018. Twenty-one aneurysms were treated with intraluminal embolization (ILE), and six were treated with parent artery occlusion (PAO) with or without bypass surgery. Radiological results, clinical outcomes and risk factors for major recurrence were assessed.

Results : The initial clinical status was similar in both groups; however, the last status was better in the ILE group than in the PAO group ( $p=0.049$ ). Neurological deterioration resulted from mass effect in one case and rupture in one after ILE, and mass effect in two and perforator infarction in one after PAO. Twenty cases (94.2\%) in the ILE group initially achieved complete occlusion or residual neck status. However, 13 cases (61.9\%) showed major recurrence, the major causes of which included coil migration or compaction. Seven cases (33.3\%) ultimately achieved residual sac status after repeat treatment. In the PAO group, all initially showed complete occlusion or a residual neck, and just one case ultimately had a residual sac. Two cases showed major recurrence, the cause of which was incomplete PAO. Aneurysm wall calcification was the only significantly protective factor against major recurrence (odds ratio, 36.12; $95 \%$ confidence interval, 1.85 to $705.18 ; p=0.018$ ).

Conclusion : Complete PAO of PTIAs is the best option if treatment-related complications can be minimized. Simple fluoroscopy is a useful imaging modality because of the recurrence pattern.

Key Words : Intracranial aneurysm · Endovascular procedures · Treatment outcome $\cdot$ Risk factors.

\section{INTRODUCTION}

Partially thrombosed intracranial aneurysms (PTIAs), characterized by organized intraluminal thrombi, are rare, and most of them are known to be large or giant sized ${ }^{6,8,9,11,12,13,20,26)}$.
PTIAs have a different natural history and are very challenging to treat, compared to typical saccular aneurysms with no intraluminal thrombus. Physicians are easily tempted to perform a simple coil embolization within a small intraluminal space. However, the natural course after the endovascular in-

- Received : July 6, 2020 •Revised : August 19, 2020 •Accepted : September 19, 2020

- Address for reprints : Won-Sang Cho

Department of Neurosurgery, Seoul National University Hospital, 101 Daehak-ro, Jongno-gu, Seoul 03080, Korea

Tel : +82-2-2072-2824, Fax : +82-2-744-8459, E-mail : nsdrcho@gmail.com, ORCID : https://orcid.org/0000-0002-3345-8718

This is an Open Access article distributed under the terms of the Creative Commons Attribution Non-Commercial License (http://creativecommons.org/licenses/by-nc/4.0) which permits unrestricted non-commercial use, distribution, and reproduction in any medium, provided the original work is properly cited. 
tervention is little known, and there are potential risks of aneurysm recurrence, growth and subsequent complications. The authors aimed to assess the radiological results and clinical outcomes of PTIAs treated with endovascular intervention and to analyze the risk factors for major recurrence.

\section{MATERIALS AND METHODS}

This study was approved by the Institutional Review Board of Seoul National University Hospital (IRB No. 1910-133-107).

\section{Patient selection}

A retrospective analysis was conducted, reviewing 34 PTIAs in 34 patients who were treated with endovascular intervention at our institute between November 1999 and March 2018 under the approval of our institutional review board. Patients followed less than 6 months after treatment $(n=3)$, those who were treated initially at other institutes $(n=2)$, those with poor medical records $(n=1)$, and those in which the intraluminal thrombus spontaneously resolved just before the intervention $(n=1)$ were excluded. Finally, 27 patients harboring 27 PTIAs were enrolled in this study. Medical records and imaging data were reviewed in terms of some parameters, including basal characteristics, radiological results, clinical outcomes, periprocedural morbidity and mortality, and risk factors for major recurrence. Clinical outcomes were evaluated using the modified Rankin Scale (mRS) ${ }^{25)}$.

The basal characteristics of the patients and aneurysms are summarized in Table 1. The mean age was $54.3 \pm 12.7$ years old (range, 11-73), and there were 11 female patients (40.7\%). Sixteen patients $(59.3 \%)$ presented with mass effect. All the PTIAs were large or giant sized, and the mean intraluminal aneurysm size was $11.7 \pm 5.3 \mathrm{~mm}$ (range, 3.0-23.7). Most PTIAs $(70 \%)$ were located in the internal carotid artery or posterior circulation. Partial or whole aneurysm wall calcification was observed in 12 cases (44.4\%).

\section{Interventional procedure and radiological evalu- ation}

All endovascular procedures were performed under general anesthesia using biplane angiographic systems such as Integris V Allura Clarity (Philips Healthcare, Best, The Netherlands) and Innova IGS 630 (General Electric Healthcare, Milwaukee,
WI, USA). PTIAs were treated with intraluminal embolization (ILE) or parent artery occlusion (PAO) with or without bypass surgery. ILE entails coil embolization within the remnant aneurysmal sac surrounded by the thrombus, and PAO entails coil embolization at both the aneurysmal sac and the parent artery harboring the aneurysm. Various types of coils, such as bare platinum or modified detachable coils, and

Table 1. Basal characteristics

\begin{tabular}{|c|c|}
\hline & Value \\
\hline No. of aneurysms/patients & $27 / 27$ \\
\hline Female : male & $11: 16$ \\
\hline Age (years) & $54.3 \pm 12.7(11-73)$ \\
\hline \multicolumn{2}{|l|}{ Presenting symptom } \\
\hline Mass effect & $16(59.3)$ \\
\hline Headache or dizziness & $10(37.0)$ \\
\hline Asymptomatic finding & $6(22.2)$ \\
\hline Seizure & $3(11.1)$ \\
\hline Rupture & $6(22.2)$ \\
\hline $\begin{array}{l}\text { Maximal diameter of aneurysm including } \\
\text { thrombus (mm) }\end{array}$ & $26.0 \pm 12.7(11.0-65.9)$ \\
\hline Small-sized (<10 mm) & 0 \\
\hline Large-sized ( $\geq 10 \mathrm{~mm}$ and <25 mm) & $14(51.9)$ \\
\hline Giant-sized ( $\geq 25 \mathrm{~mm})$ & $13(48.1)$ \\
\hline Intraluminal aneurysm size (mm) & $11.7 \pm 5.3(3.0-23.7)$ \\
\hline \multicolumn{2}{|l|}{ Location } \\
\hline Internal carotid artery & $8(29.7)$ \\
\hline Posterior communicating artery & 3 \\
\hline Anterior communicating artery & $4(14.8)$ \\
\hline Middle cerebral artery & $4(14.8)$ \\
\hline Posterior circulation & $11(40.7)$ \\
\hline Posterior cerebral artery & 3 \\
\hline Basilar artery & 3 \\
\hline Vertebral artery & 5 \\
\hline Wall calcification & $12(44.4)$ \\
\hline Partial & 6 \\
\hline Whole & 6 \\
\hline \multicolumn{2}{|c|}{$\begin{array}{l}\text { Thrombus signal intensity on } \mathrm{T} 1 \text { or TOF MR } \\
\text { imaging }\end{array}$} \\
\hline High & 16 \\
\hline Isogenous or low & 11 \\
\hline
\end{tabular}

Values are presented as mean \pm standard deviation (range) or number (\%) unless otherwise indicated. TOF : time-of-flight, MR : magnetic resonance 
stents, such as Neuroform (Stryker Neurovascular, Fremont, CA, USA) and Enterprise (Codman \& Shurtleff, Raynham, MA, USA) stents, were used according to the physicians' preference.

Antiplatelet premedication and concurrent monitoring with the VerifyNow P2Y12 assay were used for patients with unruptured aneurysms. If patients were poor responders based on the VerifyNow P2Y12 assay, additional antiplatelet medication was administered ${ }^{5}$. A bolus of heparin (3000 IU), injected intravenously upon the placement of the femoral arterial sheath, was thereafter sustained by hourly doses (1000 IU), and activated clotting time was monitored each hour. Antiplatelet premedication and a bolus of heparin were not administered in patients with ruptured aneurysms. Continued dual antiplatelet therapy was advised for at least 3 months postoperatively, followed by single-agent maintenance for at least 1 year in patients with stent assistance. In the absence of a stent, antiplatelet therapy was selectively used in cases with prior antiplatelet medication use, coil protrusion and procedural thromboembolism.

All cases were initially evaluated with magnetic resonance (MR) imaging, computed tomography and digital subtraction angiography (DSA). Follow-up radiological examination was performed with MR angiography at 6, 12, 24, and 36 months after the endovascular procedure. MR angiography (1.5 or 3 Tesla) was used for the acquisition of 3D reconstruction and source images. DSA was recommended when MR angiography was not available or when major recanalization was suspected on MR and repeat embolization was necessary. Angiographic results were graded according to the Raymond-Roy occlusion classification ${ }^{18)}$. Major recanalization was defined as a residual sac on the follow-up DSA. Angiographic results were evaluated by three experienced interpreters who were blinded to pertinent clinical and radiological information. The signal intensity of the thrombi around the central lumen on T1 or time-of-flight MR imaging was classified into high, isogenous or low, and approximately $59.3 \%$ of the thrombi showed high signal intensity at least near the intraluminal space (Table 1).

\section{Statistical analyses}

Continuous data were expressed as the mean \pm standard deviation. Fisher's exact, Wilcoxon's signed rank and the MannWhitney $\mathrm{U}$ tests were used to assess categorical or continuous variables, respectively. A univariate analysis of parameters impacting major recanalization was conducted via binary logistic regression, including age, sex, rupture, aneurysm size, lumen to whole aneurysm ratio, aneurysm location (post vs. anterior circulation), treatment method (ILE vs. PAO), initial occlusion result, the use of a stent, thrombus signal intensity on T1 or time-of-flight MR imaging (high vs. iso- or low), follow-up duration and wall calcification. Variables with $p$-values $<0.20$ in a univariate analysis were selected for the multivariate analysis to determine risk factors. Statistical significance was set at $p<0.05$, employing standard software (SPSS ver. 22; IBM, Armonk, NY, USA) for all purposes.

\section{RESULTS}

\section{Clinical outcomes}

Twenty-six patients (96.3\%) showed a favorable functional status (mRS, 0-2) just before the initial treatment, and 21 (77.8\%) achieved a favorable outcome ( $p=0.434)$. Overall, 22 $(81.5 \%)$ of 27 patients were stable or had improved. Comparing the mRS scores between the ILE and PAO groups at each period, those just before the initial treatment were statistically similar $(0.8 \pm 0.5$ [range, $0-2$ ] in the ILE group vs. $1.7 \pm 1.8$ [range, $0-5]$ in the PAO group; $p=0.239$ ). However, those at the last follow-up period were better in the ILE group than in the PAO group (0.9 \pm 1.4 [range, $0-2$ ] vs. $2.3 \pm 1.8$ [range, $0-5$ ]; $p=0.049$ ) (Supplementary Fig. 1).

In the ILE group, all patients except two showed the same neurological status between the initial and the last periods. One patient presented with a rupture of the aneurysm, which had been treated two times with ILE, and a 3rd ILE was performed. However, the neurological sequelae have still remained after aneurysmal rupture (mRS, 0 initially and 5 ultimately). The other suffered from progressive mass effect even after the ILE (mRS, 1 initially and 4 ultimately), and the patient died due to acute myocardial infarction during the hesitation of repeat neurointervention. In the PAO group, two patients showed neurological deterioration due to aneurysm growth after the incomplete PAO (mRS from 1 to 5 and from 2 to 3 , respectively), and one patient experienced cerebral infarction due to the occlusion of perforators immediately after PAO (mRS, 2 initially and 3 ultimately). The other three patients were stable or improved at the last follow-up. 
There was no mortality case related to the aneurysms. Two patients (7.4\%) transiently experienced periprocedural thromboembolic events. One patient (3.7\%) had a permanent neurological deficit due to the occlusion of the lenticulostriate artery after the trapping of the middle cerebral artery (MCA) harboring the aneurysm.

\section{Radiological results}

A total of 27 cases were treated with ILE for 21 and PAO for six. Two of 6 PAO cases underwent simultaneous bypass surgery for flow restoration in the territory of the occluded parent artery. The angiographic results are summarized in Table 2. Twenty-six aneurysms (96.3\%) achieved complete occlusion or residual neck states immediately after initial treatment. Despite repeat treatment in 12 cases, eight (29.6\%) ultimately remained with a status of residual sac at the last follow-up, with a mean follow-up duration of $53.5 \pm 48.7$ months (range, 2.9207.3).

Of 21 aneurysms initially treated with ILE, $94.2 \%(n=20)$ of the cases initially showed complete occlusion or residual neck states. However, 13 aneurysms (61.9\%) resulted in major recurrence in a mean duration of $30.7 \pm 48.7$ months (range, 4.6182.4) after the initial treatment, among which nine cases had achieved a complete occlusion, three had a residual neck, and the last one had a residual sac at first. Three cases (each one from each status of complete occlusion, residual neck and sac) were conservatively observed because of the patients' refusal, and the other 10 underwent repeat embolization. Repeat ILE was performed once in seven cases, twice in two cases and three times in one case. Even after the repeat treatment in 10 cases, $40 \%$ remained with a status of residual sac (Supplemen- tary Table 1). Ultimately, seven cases (33.3\%) still showed residual sac (mean follow-up duration of 62.8 \pm 50.6 months [range, 6.4-207.3]).

Imaging patterns of major recurrence after ILE in 13 cases included coil migration or dispersion into the thrombus in 10 (76.9\%), coil compaction in seven (53.8\%), a decrease in thrombus volume in four (30.8\%) and aneurysm growth in two (15.4\%) (Fig. 1). Only two cases showed major recurrence without coil mass change. Interestingly, in the other eight cases without major recurrence during the follow-up, there was no evidence of coil compaction or migration, and seven of eight cases were accompanied by calcification along the aneurysm wall (Supplementary Fig. 2).

Of the six aneurysms initially treated with PAO, five cases were completely occluded at first (Fig. 2). Two aneurysms (33.3\%) showed major recurrence during the follow-up : the intraluminal space of the one case just distal to the MCA bifurcation had been completely occluded, and the inferior division of the MCA had been occluded, with the superior division of the MCA remaining unoccluded; and the other case located at the vertebral artery had been incompletely occluded, with the proximal parent artery trapped, but the distal parent artery remained untrapped after the initial treatment. The cause of major recurrence was the incomplete trapping of parent arteries, and coil compaction on fluoroscopy was identified in all cases. All patients were repeatedly treated with bypass surgery and endovascular or surgical trapping of the parent arteries. Only one case (16.7\%), which was one of two cases of major recurrence, ultimately achieved residual sac status because of the retrograde filling from the distal vertebral artery, and the other five cases ultimately showed states of

Table 2. Angiographic results

\begin{tabular}{|c|c|c|c|}
\hline & Total patient $(n=27)$ & ILE $(n=21)$ & PAO $(n=6)$ \\
\hline \multicolumn{4}{|c|}{ Immediately after initial treatment } \\
\hline Complete occlusion & 15 (55.6) & $10(47.6)$ & $5(83.3)$ \\
\hline Residual neck & $11(40.7)$ & $10(47.6)$ & $1(16.7)$ \\
\hline Residual sac & $1(3.7)$ & $1(4.8)$ & $0(0.0)$ \\
\hline \multicolumn{4}{|c|}{ At the last follow-up period } \\
\hline Complete occlusion & $11(40.7)$ & $7(33.3)$ & $4(66.7)$ \\
\hline Residual neck & $8(29.6)$ & $7(33.3)$ & $1(16.7)$ \\
\hline Residual sac & $8(29.6)$ & $7(33.3)$ & $1(16.7)$ \\
\hline
\end{tabular}

Values are presented as number of cases (\%). ILE : intraluminal embolization, PAO : parent artery occlusion 

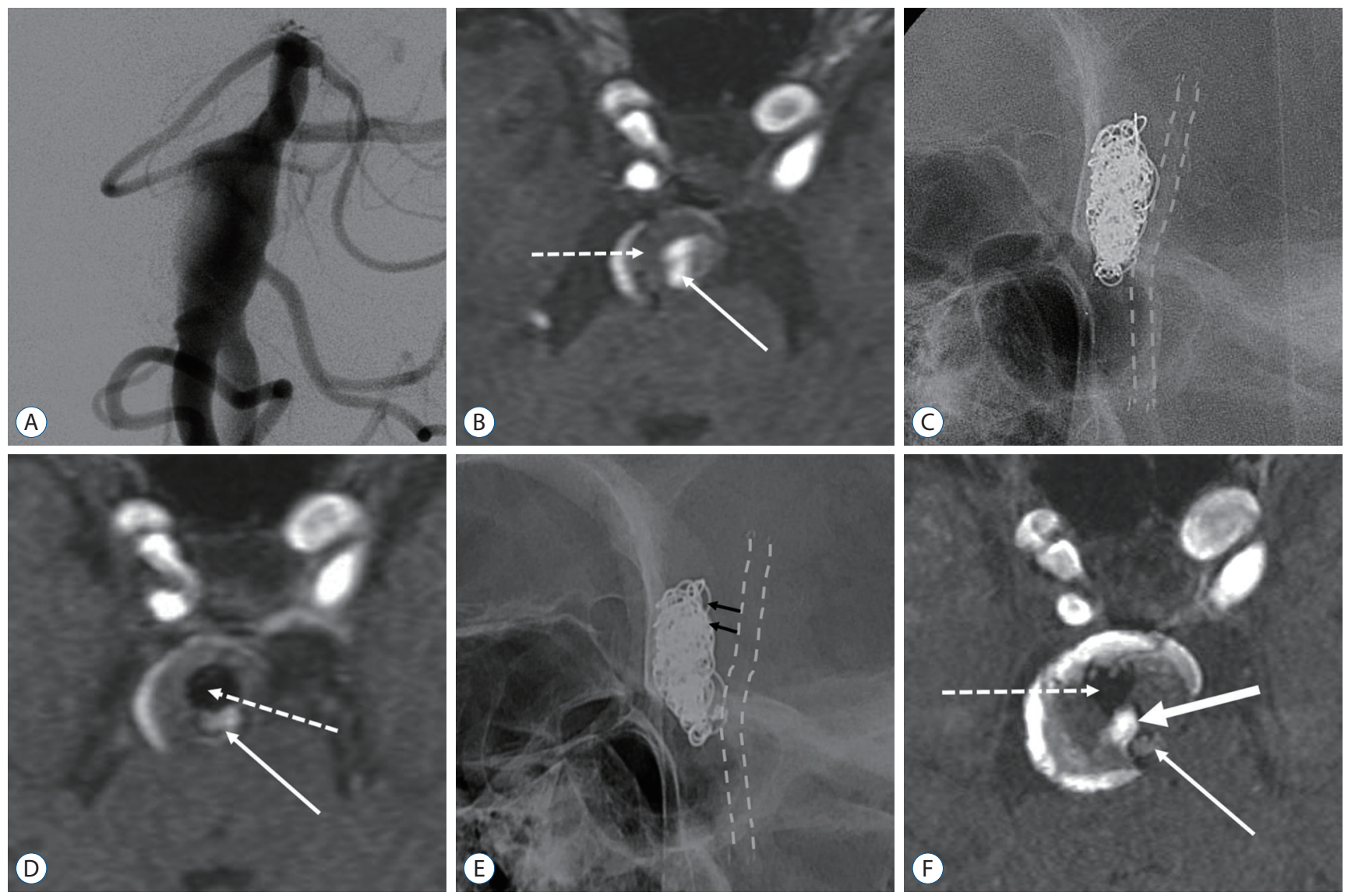

Fig. 1. An approximately 20-mm-sized thrombosed aneurysm arising from the basilar artery on cerebral angiography (A) and time-of-flight magnetic resonance angiography ( $\mathrm{B}$; arrow : basilar artery, dotted arrow : thrombus). After the initial stent-assisted intraluminal coil embolization, the coil mass and stent (dotted lines) were identified on fluoroscopy (C) and magnetic resonance imaging (D; arrow : basilar artery, dotted arrow : coil mass). During the follow-up, however, aneurysm recurrence was identified at 24 months, and aneurysm growth (approximately $28 \mathrm{~mm}$ ) and the migration of the coil

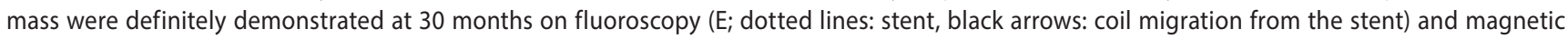
resonance imaging ( $\mathrm{F}$; arrow : basilar artery, thick arrow : recurred sac, dotted line : migrating coil mass).
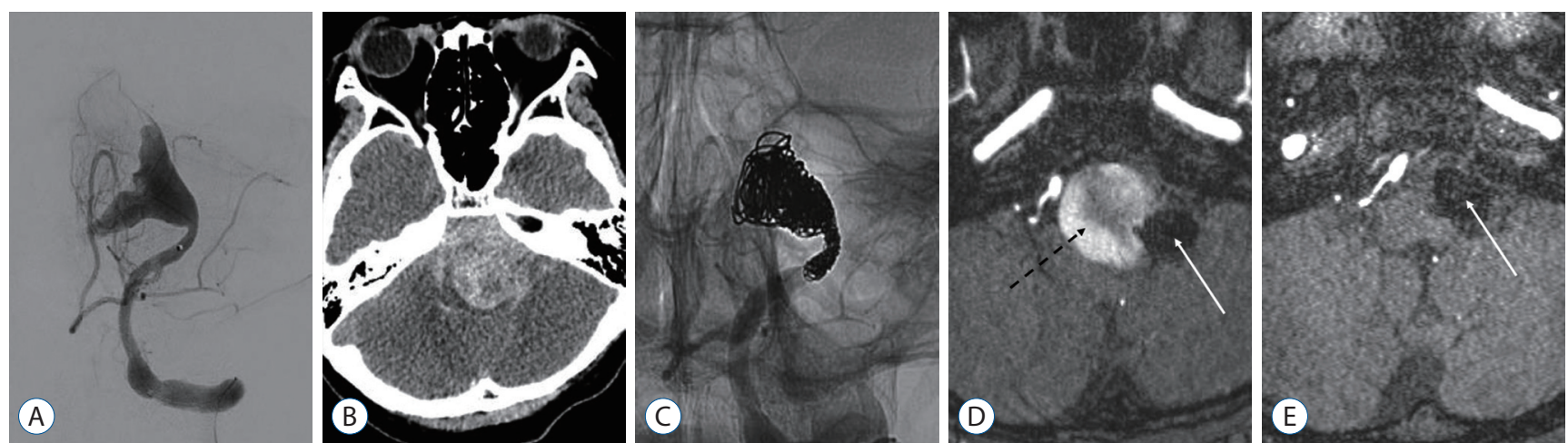

Fig. 2. An approximately $18-\mathrm{mm}$-sized thrombosed aneurysm at the left vertebral artery presenting with subarachnoid hemorrhage on cerebral angiography (A) and computed tomography (B). Parent artery occlusion (the trapping of the proximal vertebral artery and intraluminal embolization) was performed (C). Coil mass (white arrow) and intra-aneurysmal thrombus (dotted black arrow) were identified on the 1-month follow-up time-offlight magnetic resonance angiography (D). On the 14-month follow-up imaging, the thrombus was completely resolved, and a coil mass (white arrow) was observed (E). 
complete occlusion or residual neck during the mean followup period of 21.1 \pm 21.8 months (range, 2.9-63.0) (Supplementary Fig. 3).

According to the last follow-up angiography, a decrease in aneurysm size was more frequently observed in completely occluded lesions, while an increase in size was more commonly observed in lesions with residual sac status after the treatment. Complete occlusion was achieved in 11 cases : aneurysm size was stable in six cases and decreased in five cases (45.5\%). Residual neck status was shown in eight cases, seven $(87.5 \%)$ of which were stable in size and one showed a decrease in size. Residual sac status was observed in eight cases, in which three cases (37.5\%) showed an increase in aneurysm size, three were stable in size and the other two were unable to be measured because of incomplete imaging data (Supplementary Table 2).

\section{Risk factor analysis for major recurrence}

In univariate and multivariate analyses, aneurysm wall without calcification was found to be significantly associated with major recurrence in all cases (odds ratio [OR], 36.12; 95\% confidence interval $[\mathrm{CI}], 1.85$ to $705.18 ; p=0.018)$ and in the ILE group (OR, 19.02; 95\% CI, 1.55 to 233.16 ; $p=0.021$ ) (Tables

Table 3. Risk factor analysis of major recurrence after endovascular treatment in total cases

\begin{tabular}{lcc}
\hline Variable & $\boldsymbol{p}$-value & OR (95\% CI) \\
\hline Univariate analysis & & \\
Aneurysm size & 0.569 & $0.64(0.14-2.94)$ \\
Lumen/whole size ratio & 0.236 & $15.85(0.16-1536.93)$ \\
Location & 0.080 & $4.44(0.84-23.58)$ \\
Ruptured aneurysm & 0.313 & $0.38(0.06-2.52)$ \\
Treatment method & 0.077 & $8.13(0.80-82.73)$ \\
Initial occlusion result & 0.346 & $0.48(0.10-2.23)$ \\
Sex & 0.816 & $1.20(0.26-5.59)$ \\
Age & 0.218 & $1.05(0.97-1.13)$ \\
Follow-up period & 0.874 & $1.00(0.98-1.02)$ \\
Wall without calcification & 0.017 & $8.25(1.45-46.86)$ \\
Multivariate analysis & & \\
Location & 0.999 & \\
Treatment method & 0.999 & \\
Wall without calcification & 0.018 & $36.12(1.85-705.18)$ \\
\hline
\end{tabular}

OR : odds ratio, $\mathrm{Cl}$ : confidence interval
3 and 4$)$.

\section{DISCUSSION}

PTIAs are generally considered different from typical saccular aneurysms because of their morphology, symptoms such as mass effect or thromboembolism rather than rupture, and difficulty in treatment with usual strategies, ${ }^{8,10-12)}$. There are some explanations about the pathophysiology of PTIAs. Basically, aneurysmal dilatation is thought to originate from repeated subintimal dissection by intrinsic and extrinsic factors $^{14)}$. Thereafter, an intra-aneurysmal thrombus may be formed by several possible mechanisms. First, the vasa vasorum within the aneurysm wall may induce repeated intramural hemorrhage and progressive thrombus formation ${ }^{22)}$. This hypothesis was based on imaging findings such as the rim enhancement of the aneurysm wall and fresher clot near the periphery of the thrombus ${ }^{12,22,23)}$. Second, intrathrombotic capillary formation may cause repeat intramural and intrathrombotic hemorrhage and progressive thrombosis based on histological examination ${ }^{16)}$. The third possible mechanism is the hemodynamic effect that blood stagnation and clot for-

Table 4. Risk factor analysis of major recurrence after intraluminal embolization in 21 cases

\begin{tabular}{lcc}
\hline Variable & $p$-value & OR (95\% Cl) \\
\hline Univariate analysis & & \\
Aneurysm size & 0.965 & $1.04(0.17-6.40)$ \\
Lumen/whole size ratio & 0.568 & $4.28(0.03-630.62)$ \\
Location & 0.999 & \\
Ruptured aneurysm & 0.855 & $1.27(0.10-16.81)$ \\
Initial occlusion result & 0.115 & $0.21(0.03-1.47)$ \\
Use of stent & 0.335 & $0.32(0.03-3.56)$ \\
Thrombus signal on MR & 0.920 & $1.11(0.14-8.68)$ \\
Sex & 0.625 & $0.63(0.11-3.71)$ \\
Age & 0.813 & $1.01(0.92-1.11)$ \\
Follow-up period & 0.766 & $1.00(0.98-1.02)$ \\
Wall without calcification & 0.012 & $23.33(1.99-273.29)$ \\
Multivariate analysis & & \\
Initial occlusion result & 0.373 & $0.34(0.03-3.65)$ \\
Wall without calcification & 0.021 & $19.02(1.55-233.16)$ \\
\hline
\end{tabular}

$\mathrm{OR}$ : odds ratio, $\mathrm{Cl}$ : confidence interval, $\mathrm{MR}$ : magnetic resonance 
mation may occur in large or giant aneurysms ${ }^{2}$. One of our patients excluded from this study showed complete resolution of their thrombus just before the treatment.

Endovascular treatment is one of the standard modalities for cerebral aneurysms ${ }^{4,15}$. Despite its low morbidity and mortality, the major drawback of endovascular treatment is a high risk of recurrence and retreatment compared to surgical treatment ${ }^{15,19)}$. The recurrence and retreatment rates in typical nonthrombosed aneurysms range from $10 \%$ to $33.6 \%$ and from $4.7 \%$ to $12.3 \%$, respectively ${ }^{19)}$. A higher recurrence rate is reported in PTIAs. Kim and $\mathrm{Choi}^{11)}$ demonstrated that the recanalization rate in PTIAs was up to fivefold higher than that in nonthrombosed aneurysms. A systemic review revealed a higher recurrence in neurointervention than surgical treatment $(62 \% \text { vs. } 2 \%)^{9)}$. In this study, $29.6 \%$ finally showed major recurrence. If not treated, the recurrence of PTIAs can lead to progressive neurological deterioration due to growth, subsequent mass effect or rupture, and embolic infarction originating from the intra-aneurysmal thrombus. Therefore, treatment strategies for the PTIAs should be different from those of typical aneurysms.

Endovascular treatment for PTIAs consists of ILE and PAO with or without bypass surgery. According to the previous reports, complete PAO seems better than ILE and incomplete $\mathrm{PAO}$ in terms of angiographic outcomes ${ }^{8,911,20,21,26)}$. Ferns et al. ${ }^{8)}$ reported that there was no additional treatment required after PAO, while 75\% of PTIAs after ILE showed recurrence. Yang et al. ${ }^{26)}$ demonstrated that no PTIAs required retreatment after PAO. In the current study, 33.3\% of the cases treated with ILE ultimately showed major recurrence even after repeated treatment, and there were no recurrences in four initial and one repeat complete PAO. In addition, size reduction was more frequently observed in completely occluded lesions, while aneurysm growth was observed only in cases with major recurrence. Ferns et al. ${ }^{8)}$ also demonstrated that reduction in aneurysm size after PAO occurred more often than after ILE (17/18 vs. $2 / 28)$. On the other hand, clinical outcomes seem controversial. Some reports showed better clinical outcomes in the complete PAO group, and vice versa in others, including this study ${ }^{6,8,11,20)}$. Therefore, treatment techniques should be determined considering the recurrence rate and procedural risks.

It is generally accepted that angiographic results are superior and clinical outcomes are inferior in surgery in typical aneu- rysms $^{4,15)}$. In the PTIAs, however, evidence is limited that angiographic results were better in surgical treatment, and clinical outcomes remained inconclusive $e^{9,13,21,24,26)}$. Two recent systemic reviews reported a higher rate of complete exclusion in surgery than in neurointervention (approximately $94 \%$ vs. $48-65 \%)^{9,21)}$. Lawton et al. ${ }^{13)}$ demonstrated that surgery achieved complete exclusion in $97 \%$ and that clinical outcomes were satisfactory (improved or unchanged in $87 \%$ and independent in 79\%). A systemic review showed better angiographic results in surgery and similar clinical outcomes; however, all of them were located in the $\mathrm{MCA}^{21}$. A certain surgical group reported $50 \%$ of the disability even after complete exclusion $^{24)}$. Because of the high risk of treatment and the rarity of incidence in PTIAs, such controversies would exist for a while. Thus, an interdisciplinary treatment strategy would be the best option based on the factors of aneurysm, patient and institution. Recently, flow diverters have been actively attempted in the field of neurointervention, and promising results have been reported. Flow diverters could be a good alternative in the near future. There are also reports about very rare cases that should be surgically removed even after the complete occlusion of the aneurysms from the parent artery because of the progressive growth by the vasa vasorum ${ }^{10}$. Physicians should also be aware of such a rare situation in treating PTIAs.

As recurrence is very common in PTIAs after the endovascular treatment of intracranial aneurysms, imaging follow-up is very important. Although DSA, MR and computed tomography are effective as imaging tools, they have some invasiveness and limitations. Fortunately, simple fluoroscopy can be effective because of the recurrence patterns of PTIAs. In this study as well as in previous reports, the dominant patterns of recurrence are coil migration into the thrombus and coil compaction ${ }^{8,11}$. Thus, a simple X-ray would be effective, economic and safe as a follow-up imaging modality. Although there is a lack of evidence for the follow-up protocol of simple fluoroscopy, we recommend that it is performed every 6 or 12 months after the endovascular treatment. If PTIA shows stable occlusion during the follow-up period, it could be performed every 12 or 24 months.

Until now, there has been no study on the risk factors for recurrence in PTIAs after neurointervention. Generally known risk factors for recurrence after the endovascular treatment of typical cerebral aneurysms are large size, wide neck, rupture, 
location, incomplete occlusion, follow-up duration and stent $^{3,17,18)}$. Wall calcification was the only significant factor protecting for major recanalization in this study. Vascular calcification is a marker of atherosclerosis, occurring in the intimal layer of the arterial wall, commonly found in the aorta and the coronary, carotid and renal arteries. Intimal calcification represents advanced atherosclerosis formation and tissue degeneration, ${ }^{1,7)}$. Cho et al. ${ }^{6}$ suggested that mural calcification within aneurysmal thrombi indicated an organized thrombus for a substantial period. It is thought that our result may support a vasa vasorum theory about the pathophysiology of PTIAs $^{12,22)}$. PTIAs continue to grow by repeated intramural hemorrhage and thrombus formation via the vasa vasorum. When the intimal calcification starts to form, however, the vasa vasorum would degenerate, and thrombus formation would stop. Finally, previous thrombi would become hard and organized. Then, recurrence would rarely occur because it is difficult for coils to migrate into the hard, organized thrombus. In addition, repeat ILE may cure the aneurysm even when coil migration occurs because wall calcification would prevent aneurysm growth. Therefore, ILE can be a good alternative to PAO in some PTIAs with calcified walls.

The correlation between recurrence and MR signal intensity of the thrombus has not been studied. Most imaging studies have examined the pathophysiology of PTIAs in which rim enhancement represented a vascularized wall and an intraaneurysmal thrombus looked like an onion shell with fresh hemorrhage at the periphery of the thrombus near the wall and older clot near the central lumen ${ }^{12,23)}$. The authors tried to elucidate the relationship between recurrence and thrombus hardness on the assumption that recurrence might happen more frequently in lesions with a softer thrombus. However, such an assumption failed to be proven because the authors used the thrombus signal around the central lumen on the initial MR imaging, considering it as a fixed status of the whole thrombus. The thrombus at a certain period had a heterogeneous signal intensity with multiple layers. In addition, the signal intensity of the thrombus at a certain area was dynamic, changing over time. The thrombus signal intensity merely had a tendency : high near the central lumen and isogenous or low at the periphery in approximately $60 \%$ of the cases, which is contrary to the findings of previous reports ${ }^{12,23}$. It may be speculated that blood inflow from the parent artery to the lumen after recurrence by coil migration and compac- tion would cause an inflammatory reaction within the thrombus, resulting in a change in thrombus composition. Additionally, inflammatory cell invasion into the thrombus may develop via the vasa vasorum or intrathrombotic capillary channels. For such reasons, there may be a limitation in evaluating the MR signal intensity as a candidate risk factor for recurrence.

Our study has a few limitations. It was a retrospective, observational, and single-center study with a small sample size. This study focused on the endovascular treatment of PTIAs, although the surgical role is also important.

\section{CONCLUSION}

Durability was not satisfactory in the treatment of PTIAs with endovascular intervention. Comparing the two technical options of PAO and ILE, the angiographic results were better in the PAO group, and the clinical outcomes were better in the ILE group. ILE and incomplete PAO seem to be a major cause of angiographic recurrence. Neurological deterioration was caused by rupture or the mass effect of recurred aneurysms and perforator infarction after PAO. Aneurysm wall calcification was a protective factor against major recurrence. In treating PTIAs, therefore, complete PAO with or without surgical bypass can be recommended as the first treatment option if the risk of treatment-related complications can be minimized. ILE should be considered as an alternative if other options are not available or for PTIAs with a calcified wall. However, the selection of treatment modalities should consider patients' condition, the characteristics of the aneurysm and adjacent vessels, and institutional situations. Most cases with major recurrence showed coil migration into the thrombus or coil compaction. Therefore, fluoroscopy can be a useful imaging modality for follow-up.

\section{CONFLICTS OF INTEREST}

No potential conflict of interest relevant to this article was reported. 


\title{
INFORMED CONSENT
}

This type of study does not require informed consent.

\section{AUTHOR CONTRIBUTIONS}

\author{
Conceptualization : WSC
}

Data curation : JL, REY, WSC

Formal analysis : WSC

Funding acquisition : JL, WSC

Methodology: WSC, YDC, HSK

Project administration : JL, WSC

Visualization : JL, WSC

Writing - original draft : JL, REY, WSC

Writing - review \& editing : WSC, REY, DHY, YDC, HSK, JEK

\section{ORCID}

Jeongjun Lee https://orcid.org/0000-0001-6847-1130

Won-Sang Cho https://orcid.org/0000-0002-3345-8718

Roh Eul Yoo

Dong Hyun Yoo

Young Dae Cho https://orcid.org/0000-0002-5625-5921 https://orcid.org/0000-0003-1658-5341 https://orcid.org/0000-0002-5293-2761 Hyun-Seung Kang https://orcid.org/0000-0002-6957-1907 Jeong Eun Kim https://orcid.org/0000-0002-6927-3109

\section{- Acknowledgements}

This research was supported by grant of the Korea Health Technology R\&D Project through the Korea Health Industry Development Institute (KHIDI) funded by the Ministry of Health \& Welfare, Republic of Korea (grant number : HI17C1561).

\section{- Supplementary materials}

The online-only data supplement is available with this article at https://doi.org/10.3340/jkns.2020.0195.

\section{References}

1. Albanese I, Khan K, Barratt B, Al-Kindi H, Schwertani A : Atherosclerotic calcification: Wnt is the hint. J Am Heart Assoc 7 : e007356, 2018

2. Black SP, German WJ : Observations on the relationship between the volume and the size of the orifice of experimental aneurysms. J Neurosurg 17 : 984-990, 1960

3. Chalouhi N, Jabbour P, Singhal S, Drueding R, Starke RM, Dalyai RT, et al. : Stent-assisted coiling of intracranial aneurysms: predictors of complications, recanalization, and outcome in 508 cases. Stroke 44 : 1348-1353, 2013

4. Cho WS, Kim JE, Park SQ, Ko JK, Kim DW, Park JC, et al. : Korean clinical practice guidelines for aneurysmal subarachnoid hemorrhage. J Korean Neurosurg Soc 61 : 127-166, 2018

5. Cho WS, Lee J, Ha EJ, Kim KH, Lee J, Cho YD, et al. : Low-dose prasugrel vs clopidogrel-based tailored premedication for endovascular treatment of cerebral aneurysms. Neurosurgery 85 : E52-E59, 2019

6. Cho YD, Park JC, Kwon BJ, Hee Han M : Endovascular treatment of largely thrombosed saccular aneurysms: follow-up results in ten patients. Neuroradiology 52 : 751-758, 2010

7. Doherty TM, Asotra K, Fitzpatrick LA, Qiao JH, Wilkin DJ, Detrano RC, et al. : Calcification in atherosclerosis: bone biology and chronic inflammation at the arterial crossroads. Proc Natl Acad Sci USA 100 : 1120111206, 2003

8. Ferns SP, van Rooij WJ, Sluzewski M, van den Berg R, Majoie CB : Partially thrombosed intracranial aneurysms presenting with mass effect: long-term clinical and imaging follow-up after endovascular treatment. AJNR Am J Neuroradiol 31 : 1197-1205, 2010

9. Güresir $E$, Wispel $C$, Borger $V$, Hadjiathanasiou A, Vatter $H$, Schuss $P$ : Treatment of partially thrombosed intracranial aneurysms: single-center series and systematic review. World Neurosurg 118 : e834-e841, 2018

10. lihara K, Murao K, Sakai N, Soeda A, Ishibashi-Ueda H, Yutani C, et al. : Continued growth of and increased symptoms from a thrombosed giant aneurysm of the vertebral artery after complete endovascular occlusion and trapping: the role of vasa vasorum. Case report. J Neurosurg 98 : 407-413, 2003

11. Kim SJ, Choi IS : Midterm outcome of partially thrombosed intracranial aneurysms treated with guglielmi detachable coils. Interv Neuroradiol 6 : 13-25, 2000

12. Krings $T$, Alvarez $H$, Reinacher $P$, Ozanne $A$, Baccin $C E$, Gandolfo $C$, et al. : Growth and rupture mechanism of partially thrombosed aneurysms. Interv Neuroradiol 13 : 117-126, 2007

13. Lawton MT, Quiñones-Hinojosa A, Chang EF, Yu T : Thrombotic intracranial aneurysms: classification scheme and management strategies in 68 patients. Neurosurgery $56:$ 441-454, 2005

14. Mizutani T, Miki Y, Kojima H, Suzuki H : Proposed classification of nonatherosclerotic cerebral fusiform and dissecting aneurysms. Neurosurgery 45 : 253-259, 1999

15. Molyneux A, Kerr R, Stratton I, Sandercock P, Clarke M, Shrimpton J, et al. : International subarachnoid aneurysm trial (ISAT) of neurosurgi- 
cal clipping versus endovascular coiling in 2143 patients with ruptured intracranial aneurysms: a randomised trial. Lancet 360 : 1267-1274, 2002

16. Nagahiro S, Takada A, Goto S, Kai Y, Ushio Y : Thrombosed growing giant aneurysms of the vertebral artery: growth mechanism and management. J Neurosurg 82 : 796-801, 1995

17. Nishido H, Piotin M, Bartolini B, Pistocchi S, Redjem H, Blanc R : Analysis of complications and recurrences of aneurysm coiling with special emphasis on the stent-assisted technique. AJNR Am J Neuroradiol 35 : 339-344, 2014

18. Raymond J, Guilbert F, Weill A, Georganos SA, Juravsky L, Lambert A, et al. : Long-term angiographic recurrences after selective endovascular treatment of aneurysms with detachable coils. Stroke 34 : 1398-1403, 2003

19. Ries T, Siemonsen S, Thomalla G, Grzyska U, Zeumer H, Fiehler J : Longterm follow-up of cerebral aneurysms after endovascular therapy prediction and outcome of retreatment. AJNR Am J Neuroradiol 28 : 17551761, 2007

20. Roccatagliata L, Guédin P, Condette-Auliac S, Gaillard S, Colas F, Boulin A, et al. : Partially thrombosed intracranial aneurysms: symptoms, evolution, and therapeutic management. Acta Neurochir (Wien) 152 : 2133-2142, 2010
21. Scerrati A, Sabatino G, Della Pepa GM, Albanese A, Marchese E, Puca A, et al. : Treatment and outcome of thrombosed aneurysms of the middle cerebral artery: institutional experience and a systematic review. Neurosurg Rev 42 : 649-661, 2019

22. Schubiger 0 , Valavanis $A$, Wichmann $W$ : Growth-mechanism of giant intracranial aneurysms; demonstration by CT and MR imaging. Neuroradiology 29 : 266-271, 1987

23. Teng MM, Nasir Qadri SM, Luo CB, Lirng JF, Chen SS, Chang CY : MR imaging of giant intracranial aneurysm. J Clin Neurosci $10: 460-464$, 2003

24. Uede T, Ohtaki M, Tanabe S, Hashi K : Direct surgical management of giant and large intracerebral aneurysms, associated with intraluminal thrombus and/or atherosclerotic thickening of aneurysmal neck. No Shinkei Geka 25 : 1007-1015, 1997

25. Uyttenboogaart M, Stewart RE, Vroomen PC, De Keyser J, Luijckx GJ : Optimizing cutoff scores for the Barthel index and the modified Rankin scale for defining outcome in acute stroke trials. Stroke 36 : 19841987, 2005

26. Yang K, Park JC, Ahn JS, Kwon DH, Kwun BD, Kim CJ : Characteristics and outcomes of varied treatment modalities for partially thrombosed intracranial aneurysms: a review of 35 cases. Acta Neurochir (Wien) 156 : 1669-1675, 2014 